\title{
Life satisfaction and positive and negative feelings of workers: a systematic review protocol
}

\author{
Viviane Cruvinel Di Castro ${ }^{*}$, Janete Capel Hernandes, Mauro Elias Mendonça and Celmo Celeno Porto
}

\begin{abstract}
Background: In this article, conceptualization of well-being is a starting point. According to Diener, subjective wellbeing refers to all kinds of evaluation, both positive and negative, people make about their own lives. It includes cognitive assessments, such as satisfaction with life and satisfaction with work, as well as affective reactions to life events, such as sadness and contentment. Low levels of health and well-being in workers lead to many consequences. Sick leave, low productivity, and absenteeism are some examples. In this systematic review, the main objective is to assess workers' subjective well-being.

Methods: The studies should include workers, whether they are paid or volunteers. Also, they must assess workers' subjective well-being. Observational peer-reviewed studies will be included. Qualitative studies will be excluded. The primary outcomes to be considered are the subjective well-being indicators described. Only studies that used six (6) instruments, developed by Diener, will be included. The instruments are Satisfaction With Life Scale (SWLS), Scale of Positive and Negative Experience (SPANE), Positive Thinking Scale (PTS), Flourishing Scale (FS), Comprehensive Inventory of Thriving (CIT), and Brief Inventory of Thriving (BIT). The studies will come from Scientific Electronic Library Online (SciELO), Biblioteca Virtual em Saúde (BVS), Portal da Coordenação de Aperfeiçoamento de Pessoal de Nível Superior (CAPES), MEDLINE, Embase, Web of Science, and Global Index Medicus databases. The studies must be written in Portuguese, English, or Spanish.

Discussion: As far as we know, this is the first systematic review related specially to workers' subjective well-being. We hope that this study contributes to the "well-being at work" discussion and also to the development of effective interventions, used outside and inside organizations, that could improve well-being scores and increase correlate variables scores such as general health, social relations, and quality of life.
\end{abstract}

Systematic review registration: PROSPERO CRD42016039520

Keywords: Subjective well-being, Worker, Systematic review, Protocol

\section{Background}

In this article, conceptualization of well-being is a starting point. The article published by Diener in 1984, under the title "Subjective Well-Being," shows that components and measures of subjective well-being were subjects of research for more than a decade and were therefore undergoing changes, assessments, and evolutions [1].

\footnotetext{
*Correspondence: viviane.di@gmail.com

Faculdade de Medicina. Programa Ciências da Saúde, Universidade Federal de Goiás, Secretaria - 1a s/n - Setor Universitário, CEP 74.605-020, Goiânia, Goiás, Brazil
}

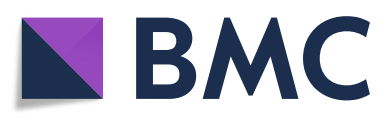

(c) The Author(s). 2018 Open Access This article is distributed under the terms of the Creative Commons Attribution 4.0 International License (http://creativecommons.org/licenses/by/4.0/), which permits unrestricted use, distribution, and reproduction in any medium, provided you give appropriate credit to the original author(s) and the source, provide a link to the Creative Commons license, and indicate if changes were made. The Creative Commons Public Domain Dedication waiver (http://creativecommons.org/publicdomain/zero/1.0/) applies to the data made available in this article, unless otherwise stated.
Research on subjective well-being has increased sigyear surrounding the topic. There are five main recent findings: multidimensionality of subjective well-being, circumstances influencing it, cultural differences, health and social relation benefits, and interventions to increase subjective well-being [2].

According to Diener, subjective well-being refers to all kinds of evaluation, both positive and negative, people make about their own lives. It includes cognitive assessments, such as satisfaction with life and satisfaction with work, as well as affective reactions to life events, such as 
sadness and contentment. Thereby, subjective well-being can be seen under two distinctive angles: one affective or emotional, represented by positive and negative feelings, and other cognitive which corresponds to life satisfaction [3]. This concept can be applied in any condition, including workers.

According to Danna and Griffin [4], low levels of health and well-being in workers lead to many consequences. Sick leave, low productivity, and absenteeism are some examples and are part of the routine of public and private companies. Corporate employee assistance programs and major lifestyle changes are some of the interventions that can promote improvements in the health and well-being of the people who work.

The concept of subjective well-being published by Diener will be fundamental in the accomplishment of this systematic review. As pointed out in one review of self-report well-being measures [5], Diener's subjective well-being model is among the most referenced theories in well-being studies.

A rationale for this review is to provide evidence that shows that Diener's tools are preferable on the evaluation of subjective well-being when compared to other tools, especially because of their psychometric properties [6].

Given the diversity of concepts and scientific theories of subjective well-being, the authors decided to use the concept assumed by Diener and the instruments developed by him. Thus, the authors of the protocol assume only one concept and can solve a possible problem of the systematic review related to the impossibility of comparison and analysis of included studies due to the diversity of instruments and associated concepts.

Another essential term and not less important is the word worker. Thus, the present systematic review will aim to assess the subjective well-being of workers based on published research on the subject.

It is known that there are systematic reviews, concluded or ongoing, dedicated to study subjective well-being and its relation to non-labor factors such as personality traits, leisure, physical exercises [7-15], and labor factors such as income and job satisfaction [16, 17]. However, until now, the authors of this protocol are unaware of any systematic review that addresses the subjective well-being of workers. Therefore, this will be the first systematic review devoted to this topic, and it will also be an opportunity to evaluate the quality of primary studies conducted on workers' well-being.

\section{Objectives}

We will perform a systematic review of studies exploring subjective well-being of workers. The study aims to answer the following research questions: (1) What is the level of workers' subjective well-being? (2) Are there differences between different types of workers?
Thereby, and more specifically, the objectives of this systematic review are:

1. Assess life satisfaction and positive and negative feelings of workers

2. Compare subjective well-being (life satisfaction and feelings) of workers of different professional categories, if applicable

\section{Methods}

The review was recorded in PROSPERO [18] database (registration number CRD42016039520). This protocol was structured according to the Preferred Reporting Items for Systematic Review and Meta-Analysis Protocols (PRISMA-P) [19] guidance [see Additional file 1].

\section{Eligibility criteria}

Studies will be selected according to the criteria outlined below.

\section{Participants}

The studies should include workers, whether they are paid or voluntary workers. In order to be qualified as a worker, participants must have a formal relationship with the company where they work (contract, worker's number), regardless of their occupational category, and they must be employed at the time the studies were performed. Retirees will be excluded. Also, the studies must assess workers' subjective well-being.

\section{Study designs}

Observational peer-reviewed studies will be included. Gray literature (such as theses, dissertations, books, and papers presented in congresses) will also be considered. Qualitative studies will be excluded. The authors of the protocol decided that the qualitative studies found in the searches will be used in a second study.

\section{Interventions or exposure}

Not applicable.

\section{Comparators}

A comparison will be made between different types of workers based on the International Standard Classification of Occupations (ISCO) [20], if applicable.

\section{Primary outcomes}

The primary outcomes to be considered are the subjective well-being indicators described in the studies.

Since the number of instruments to evaluate such a construct is very broad, and we will consider the concept of subjective well-being described by Diener in this systematic review, only studies that used six (6) instruments, also developed by Diener to evaluate subjective 
well-being, will be included. They are Satisfaction With Life Scale (SWLS) [21], Scale of Positive and Negative Experience (SPANE) [6], Flourishing Scale (FS) [6], Positive Thinking Scale (PTS) [22], Comprehensive Inventory of Thriving (CIT) [23], and Brief Inventory of Thriving (BIT) [23].

If the study has more than one variable being evaluated, only those studies that provide "subjective well-being" results separately will be considered for the purpose of this review.

We intend to divide the studies into groups for analysis purposes. Comparisons will be made between studies using the same tool to measure the same outcome. For example, a study that used SWLS to evaluate life satisfaction will have its results compared to other studies that have also used SWLS to evaluate life satisfaction. Together, such studies will be considered a group and the comparison will be made in this specific variable level.

\section{Secondary outcomes}

Secondary outcomes will be not considered in this review.

\section{Search methods}

Literature search strategies will be developed using Medical Subject Headings (MeSH), Descritores em Ciências da Saúde (DeCS), Embase Thesaurus (Emtree), and text words. We will search MEDLINE [24], Scientific Electronic Library Online (SciELO) [25], Biblioteca Virtual em Saúde (BVS) [26] database, Coordenação de Aperfeiçoamento de Pessoal de Nível Superior - CAPES [27] data, Embase [28], Web of Science [29], and Global Index Medicus [30]. The data will be reached from inception until December 15, 2018.

To ensure literature saturation, we will scan the reference lists of included studies or relevant reviews identified through the search. For searches in the databases, the following keywords or search terms will be used: "subjective well-being," "worker," "work," "occupation," "employee," "volunteer," "voluntary workers," "satisfaction with life," and "positive and negative feelings."

The data found in the search process requires the use of EndNote X9 for this process step. If repeated studies are identified, they will be removed from the research. Only studies in Portuguese, English, and Spanish will be accepted. An example of the final research strategy used for the MEDLINE database is provided [see Additional file 2]. A search in four databases indicating initial results can be seen in Table 1 .

\section{Selection of studies}

Two reviewers will independently screen the studies identified by the searches following a three-phase procedure. After each phase, the reviewers will check
Table 1 Number of studies per base

\begin{tabular}{ll}
\hline Database & Results \\
\hline Medline & 11,774 \\
BVS & 1551 \\
SCiELO & 81 \\
CAPES & 506,068 \\
Total & 519,474 \\
\hline
\end{tabular}

SCiELO Scientific Electronic Library Online, BVS Biblioteca Virtual em Saúde, CAPES Coordenação de Aperfeiçoamento de Pessoal de Nível Superior

inclusions and exclusions, and in case of disagreements, a third reviewer will be involved as an adjudicator.

For phase 1, titles of the articles identified by the searches will be screened. If repeated studies are identified, they will be removed from the research.

For phase 2, abstracts of articles will be screened against the following criteria:

1. Is the study related to subjective well-being? (yes, not clear, or no)

2. Is the study with workers? (yes, not clear, or no)

For phase 3, full texts of the studies selected on phase 2 will be read and screened against the following criteria:

1. Is it a quantitative study? (yes, not clear, or no)

2. Is the study in Portuguese, English, or Spanish? (yes, not clear, or no)

3. Did the study use any instrument developed by Diener to evaluate participants' subjective wellbeing? (yes, not clear, or no)

4. Did the study provide "subjective well-being" results separately? (yes, not clear, or no)

After that, data of the studies meeting all the above inclusion criteria will be used to elaborate a table with the following information:

1. Authors' names

2. Date of the study publication

3. Country of origin

4. Study design

5. Summary of studies containing the following information: objective, sample size, type of work (professional categories, voluntary work, nonvoluntary work), well-being variables, instruments, results, and conclusions

Reasons for the studies exclusion will be recorded. None of the review authors will be blind to the journal titles, authors, or study institutions. A flow diagram of the study will be done containing measures, such as 
identification, screening, eligibility, and inclusion and an explanatory statement on the grounds of exclusion.

\section{Data extraction and management}

Reviewers will extract data independently from each eligible study. Data abstracted will include demographic information, variables, and outcomes.

Age and gender are examples of demographic variables. Both will be extracted in mean and proportion values, respectively.

In order to avoid overlapping reports, multiple reports of a single study will be identified and excluded.

\section{Quality assessment}

To assess the risk of bias in studies, JBI Critical Appraisal Checklist tools, presented by International Joanna Briggs Institute in 2017, will be used [31].

If the studies are evaluated with an indication of high risk of bias in more than half of the items proposed by the tool, it will be considered a study with high risk of bias. If the studies are evaluated with indication of high risk of bias in half or less than half of the items of the tool, it will be considered with medium or low risk of bias.

Grading of Recommendations Assessment, Development and Evaluation (GRADE) [32] approach will be used for describing the quality of relevant evidence, if applicable.

\section{Evidence synthesis}

A systematic narrative synthesis will be provided with information presented in the text and tables to summarize and explain the characteristics and well-being results of the included studies. The narrative synthesis will explore the relationship and findings both within and between the included studies.

If there is a very high heterogeneity between studies, metanalysis will not be performed. High heterogeneity will be determined on the basis of type of work, age of participants, proportion of males/females, and type of organization. In case of homogeneity, metanalysis will be conducted.

For metanalysis, heterogeneity will be assessed using the $I^{2}$ based on the Q statistic of Cochran test. To determine the level of heterogeneity, Cochrane classification for $I^{2}$ values will be used. If $I^{2}$ is $50 \%$ or less, there is little heterogeneity. If $I^{2}$ value is between 51 and $75 \%$, there is medium heterogeneity. If $I^{2}$ is above $75 \%$, there is high heterogeneity between the included studies [33].

Heterogeneity will be explained by subgroup analysis using the following variables: age, gender, study design, study language, instruments used to evaluate subjective well-being, type of work, type of organization, risk of study bias, and method of analysis of the results.

Outcomes will be analyzed using raw mean differences. The results will be presented on a forest plot.
Metanalyses will be conducted by using random-effects method (DerSimonian and Laird), because confidence intervals for the average intervention effect will be wider and corresponding claims of statistical significance will be more conservative [34-36].

Three different tools will be used to assess meta-biases such as publication bias and outcome reporting bias. If 10 or more studies are available, the potential for publication bias will be explored through funnel plots. Additionally, Begg and Mazumdar's test [37] and Egger's test [38] will be used to assess small study effects. Also, according to the tests, if $p<0.05$, publication bias will be detected. If $p \geq 0.05$, there will be no publication bias.

Finally, for completing metanalyses, RevMan 5.3 will be used [39].

\section{Amendments to protocol}

Any substantive amendments to this protocol will be registered with PROSPERO as they occur and documented in the final publication.

\section{Dissemination}

We will publish review results in an international peer-reviewed journal and will report results according to the Preferred Reporting Items for Systematic Reviews and Meta-Analyses (PRISMA) statement.

We will also disseminate results to the research community and relevant key stakeholders through presentations at relevant academic and non-academic meetings and via social media. If findings are found to be interesting to the wider public, we will disseminate them via mass media.

\section{Discussion}

As far as we know, this is the first systematic review related specially to workers' subjective well-being. The evidence synthesis of the review will probably aid in the comparison of well-being results. We hope that this study contributes to the "well-being at work" discussion and also to the development of effective interventions, used outside and inside organizations, that could improve well-being scores and increase correlate variables scores such as general health, social relations, and quality of life.

In addition, the research will be part of a doctoral thesis, articles, posters, and discussions which may instigate further discussions on the subject in the academic community and, consequently, the preparation of proposals and guidelines designed to enhance subjective well-being of workers in general.

This systematic review may present some potential limitations. One of them may be the fact that we will not include studies that were presented at scientific events and have not been published in scientific journals. 
Also, studies in languages other than English, Portuguese, and Spanish will not be included.

At the time of data analysis, we may have problems with the lack of homogeneity of the studies. This has been a difficulty for reviewers when undertaking systematic reviews of the literature. The challenge of drawing conclusions based on very diverse studies and samples is enormous and may hamper the generalization of results.

\section{Additional files}

Additional file 1: Preferred Reporting Items for Systematic Reviews and Meta-Analysis Protocols (PRISMA-P) 2015 checklist. (DOC 86 kb)

Additional file 2: Search strategies MEDLINE. Medline search strategy. (DOCX $13 \mathrm{~kb})$

\section{Abbreviations}

BIT: Brief Inventory of Thriving; BVS: Biblioteca Virtual emSaúde; CAPES: Coordenação de Aperfeiçoamento de Pessoal de Nível Superior: Cl: Confidence intervals; CIT: Comprehensive Inventory of Thriving; DeCS: Descritores em Ciências da Saúde; Emtree: Embase Thesaurus; FS: Flourishing Scale; ISCO: International Standard Classification of Occupations; MeSH: Medical Subject Headings; PRISMA: Preferred Reporting Items for Systematic Reviews and Meta-Analyses; PRISMA-P: Preferred Reporting Items for Systematic Review and Meta-Analysis Protocols; PTS: Positive Thinking Scale; SciELO: Scientific Electronic Library Online; SPANE: Scale of Positive and Negative Experience; SWLS: Satisfaction With Life Scale

\section{Acknowledgements}

Not applicable

\section{Funding}

This study was supported by the Coordenação de Aperfeiçoamento de Pessoal de Nivel Superior (CAPES), through a research grant awarded to the first author (1614350).

Non-financial sources include the provision of library and data base by Universidade Federal de Goiás in Goiânia-GO-Brazil.

\section{Availability of data and materials}

Not applicable

\section{Authors' contributions}

VCDC and JCH drafted the protocol, search strategy, the manuscript, and registration of the systematic review. MEM contributed to the conception of the research questions and protocol, critically reviewed and provided comments on the manuscript drafts, and agreed on the final submitted version. CCP critically reviewed and provided comments on the manuscript draft and agreed on the final submitted version. All authors read, provided feedback, and approved the final manuscript.

\section{Authors' information}

Not applicable

Ethics approval and consent to participate

Not applicable

\section{Consent for publication}

Not applicable

\section{Competing interests}

The authors declare that they have no competing interests.

\section{Publisher's Note}

Springer Nature remains neutral with regard to jurisdictional claims in published maps and institutional affiliations.
Received: 27 April 2018 Accepted: 4 December 2018

Published online: 23 December 2018

\section{References}

1. Diener E. Subjective well-being. Psychol Bull. 1984;95(3):542-75.

2. Diener E, Heintzelman SJ, Kushlev K, Tay L, Wirtz D, Lutes LD, Oishi S. Findings all psychologists should know from the new science on subjective well-being. Canadian Psychology Psychologie Canadienne. 2017;58:87-104.

3. Diener E. Guidelines for national indicators of subjective well-being and illbeing. Journal of Happiness Studies: An Interdisciplinary Forum on Subjective Well-Being. 2006;7(4):397-404.

4. Danna K, Griffin RW. Health and well-being in the workplace: a review and synthesis of the literature. J Manag. 1999;25(3):357-84.

5. Linton M, Dieppe P, Medina-Lara A. Review of 99 self-report measures for assessing well-being in adults: exploring dimensions of well-being and developments over time. BMJ Open. 2016;6:e010641. https://doi.org/10. 1136/bmjopen-2015-010641.

6. Diener E, Wirtz D, Tov W, Kim-Prieto C, Choi D, Oishi S, et al. New measures of well-being: flourishing and positive and negative feelings. Soc Indic Res. 2009:39:247-66

7. DeNeve KM, Cooper $\mathrm{H}$. The happy personality: a meta-analysis of 137 personality traits and subjective well-being. Psychol Bull. 1998;124:197-229.

8. Kuykendall L, Tay L, Ng V. Leisure engagement and subjective well-being: a meta-analysis. Psychol Bull. 2015;141:364-403.

9. Pinquart $M$, Sörensen S. Influences of socioeconomic status, social network, and competence on subjective well-being in later life: a meta-analysis. Psychol Aging. 2000;15:187-224.

10. Oldervoll LM, Kaasa S, Hjermstad M, Lund JA, Loge JH. Physical exercise results in the improved subjective well-being of a few or is effective rehabilitation for all cancer patients. Eur J Cancer. 2004;40(7):951-62.

11. Martin Ginis KA, Jetha A, Mack DE, Hetz S. Physical activity and subjective well-being among people with spinal cord injury: a meta-analysis. Spinal Cord. 2010;48(1):65-72.

12. Jing $N$, Jun L. Relationships between subjective well-being and several aspects of current life circumstances: a systematic review and meta analysis. PROSPERO 2017 CRD42017057921.http://www.crd.york.ac.uk/PROSPERO/ display record.phplD=CRD42017057921 Accessed 20 Apr 2018.

13. Catharina B, Ross W, Christopher D. Systematic review of predictors of subjective well-being and quality of life of migrant populations in high-income countries. PROSPERO 2018 CRD42018087197.http://www.crd.york.ac.uk/ PROSPERO/display_record.phplD=CRD42018087197 Accessed 20 Apr 2018.

14. Ngamaba $\mathrm{KH}$, Panagioti $\mathrm{M}$, Armitage $\mathrm{CJ}$. How strongly related are health status and subjective well-being? Systematic review and meta-analysis. Eur J Pub Health. 2017:27(5):879-85.

15. Read S, Grundy E, Foverskov E. Socio-economic position and subjective health and well-being among older people in Europe: a systematic narrative review. Aging Ment Health. 2016;20(5):529-42.

16. Bowling NA, Eschleman KJ, Wang $\mathrm{Q}$. A meta-analytic examination of the relationship between job satisfaction and subjective well-being. J Occup Organ Psychol. 2010:83:915-34.

17. Ngamaba KH, Panagioti M, Armitage CJ. Income inequality and subjective wellbeing: a systematic review and meta-analysis. Qual Life Res. 2018;27(3):577-96.

18. PROSPERO - International prospective register of systematic reviews. http:// www.crd.york.ac.uk/PROSPERO/. Accessed 20 Apr 2018.

19. Shamseer $L$, Moher $D$, Clarke $M$, Ghersi D, Liberati A, Petticrew $M$, Shekelle $P$, Stewart LA. Preferred reporting items for systematic review and metaanalysis protocols (PRISMA-P) 2015: elaboration and explanation. BMJ. 2014. https://doi.org/10.1136/bmj.g7647.

20. International Standard Classification of Occupations (ISCO). http://www. ilo.org/public/english/bureau/stat/isco/docs/resol08.pdf. Accessed 08 Sept 2018.

21. Kobau R, Sniezek J, Zack MM, Lucas RE, Burns A. Well-being assessment: an evaluation of well-being scales for public health and population estimates of well-being among US adults. Appl Psychol Health Well-Being. 2010;2(3): 272-97. https://doi.org/10.1111/j.1758-0854.2010.01035.x.

22. Diener E. Assessing well-being: the collected works of Ed Diener. Social Indicators Research Series. 2009; 39 [acesso em 20 mar 2018]. Disponível em: https://www.springer.com/br/book/9789048123537.

23. Su R, Tay L, Diener E. The development and validation of Comprehensive Inventory of Thriving (CIT) and Brief Inventory of Thriving (BIT). Appl Psychol Health and Well-Being. 2014;6(3):251-79. 
24. PubMed - Us National Library of Medicine National Institutes of Health. https://www.ncbi.nlm.nih.gov/pubmed. Accessed 20 Apri 2018.

25. Scientific Electronic Library Online (SCIELO). http://scielo.org/php/index.php. Accessed 20 Apr 2018.

26. Biblioteca Virtual em Saúde (BVS). http://bvsalud.org/. Accessed 20 Apr 2018.

27. Coordenação de Aperfeiçoamento de Pessoal de Nível Superior - (CAPES). http://www.periodicos.capes.gov.br/ Accessed 20 Apr 2018.

28. Embase - Biomedical Evidence is Essential. https://www.elsevier.com/ solutions/embase-biomedical-research. Accessed 20 Apr 2018.

29. Web of Science. http://login.webofknowledge.com/error/Error?Error= IPError\&PathInfo=\%2F\&RouterURL=http\%3A\%2F\%2Fwww.webofknowledge. com\%2F\&Domain=. webofknowledge.com\&Src=IP\&Alias=WOK5. Accessed 8 Sept 2018.

30. Global Index Medicus. http://www.globalhealthlibrary.net/php/index. php?lang=en. Accessed 8 Sept 2018.

31. Moola S, Munn Z, Tufanaru C, Aromataris E, Sears K, Sfetcu R, et al. Chapter 7: Systematic reviews of etiology and risk. In: Aromataris E, Munn Z (Editors). Joanna Briggs Institute Reviewer's Manual. The Joanna Briggs Institute. https://reviewersmanual.joannabriggs.org/. Accessed 10 July 2017.

32. GRADEpro. GRADE WorkingGroup. https:/gradepro.org/. Accessed 20 Apr 2018.

33. Rodrigues C, Ziegelmann P. Metanálise: um guia prático. Revista HCPA. 2010:30(4):436-47.

34. Higgins JP, Thompson SG. Quantifying heterogeneity in a meta-analysis. Stat Med. 2002;21(11):1539-58

35. Higgins JP, Thompson SG, Deeks JJ, Altman DG. Measuring inconsistency in meta-analyses. BMJ. 2003;327(7414):557-60.

36. Borenstein M, Hedges LV, Higgins JPT, Rothstein HR. Introduction to metaanalysis. West Sussex, UK: Wiley; 2009.

37. Begg CB, Mazumdar M. Operating characteristics of a rank correlation test for publication bias. Biometrics. 1994;50(4):1088-101. https://doi.org/10. 2307/2533446

38. Egger $M$, Smith GD, Schneider M, Minder C. Bias in meta-analysis detected by a simple, graphical test. Br Med J. 1997;315(7109):629-34. dx.doi.org. https://doi.org/10.1136/bmj.315.7109.629.

39. Cochrane Collaboration. Review manager (RevMan) [computerprogram]. http://community.cochrane.org/tools/review-production-tools/revman-5. Accessed 20 Apr 2018

Ready to submit your research? Choose BMC and benefit from:

- fast, convenient online submission

- thorough peer review by experienced researchers in your field

- rapid publication on acceptance

- support for research data, including large and complex data types

- gold Open Access which fosters wider collaboration and increased citations

- maximum visibility for your research: over $100 \mathrm{M}$ website views per year

At $\mathrm{BMC}$, research is always in progress.

Learn more biomedcentral.com/submissions 